OPEN

SUBJECT AREAS:

CELLULAR

NEUROSCIENCE

AXON AND DENDRITIC

GUIDANCE

CELLULAR IMAGING

FLUORESCENCE IMAGING

Received

22 May 2013

Accepted

17 October 2013

Published

1 November 2013

Correspondence and requests for materials should be addressed to K.O. (oka@bio.keio. ac.jp)

\section{Crosstalk between Second Messengers Predicts the Motility of the Growth Cone}

\author{
Takahiko Kobayashi, Fumiaki Nagase, Kohii Hotta \& Kotaro Oka
}

Center for Biosciences and Informatics, School of Fundamental Sciences and Technology, Keio University, 3-14-1 Hiyoshi, Kohokuku, Yokohama, Kanagawa, Japan.

Axon guidance involves multiple second messenger signal transduction pathways. Although each signal transduction pathway has been characterized, only a few studies have examined crosstalk between these cascades. Here, we applied a simultaneous second messenger imaging method to the growth cone and demonstrated correlations between cAMP, cGMP, and $\mathrm{Ca}^{2+}$. The levels of cAMP and cGMP in non-stimulated freely extending growth cones showed a negative correlation without delay. Although there was no direct correlation between cAMP and $\mathrm{Ca}^{2+}$, examination of cross correlations using small time windows showed frequent switching behavior from negative to positive and vice versa. Furthermore, spatially asymmetric cAMP and cGMP signals in freely deviating growth cones were visualized directly. These results indicate that we succeed in relating second messenger crosstalk to growth cone deviation and extension, and also indicate the possibility of predicting axon guidance from this second messenger crosstalk.

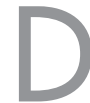

uring neural circuit formation, the growth cone navigates axons toward their targets via interactions with various guidance cues ${ }^{1,2}$. These cues activate receptors at axonal tips and change the levels of second messengers $\left(\mathrm{Ca}^{2+}, \mathrm{cAMP}\right.$, and cGMP) that play crucial roles in axon pathfinding ${ }^{3,4}$. Imaging and optical tools have revealed the importance of spatially asymmetric $\mathrm{Ca}^{2+}$ and cAMP elevation in the growth cone for the turning response ${ }^{5}$. Previous reports suggested second messenger interactions in the growth cone and neurite; cAMP levels affect $\mathrm{Ca}^{2+}$ spike frequency ${ }^{6,7}$ and $\mathrm{cAMP}$ responses to stimuli mirror those of cGMP ${ }^{8}$. Moreover, the turning responses of the growth cone to the attractive guidance cue netrin 1 depend on the cAMP:cGMP ratio?. Although cAMP, cGMP, and $\mathrm{Ca}^{2+}$ are key players in axon guidance, crosstalk between these second messengers in the growth cone has not been analyzed directly. Recent studies have reported advanced fluorescent imaging techniques using genetic tags and fluorescence resonance energy transfer (FRET) ${ }^{10-12}$. Therefore, to visualize cAMP, cGMP, and $\mathrm{Ca}^{2+}$ dynamics in the growth cone, we performed dual FRET measurements with a single excitation light using Epac1-camps ${ }^{13}$, red cGES-DE5 ${ }^{11}$, and SapRC2.12 $2^{11}$, respectively.

\section{Results}

A confocal microscope-based dual FRET system was set up (Supplementary Fig. 1). Image processing and analysis, including linear unmixing, region of interest (ROI) extraction, and ROI-by-ROI FRET ratio calculations, were executed semiautomatically using original image analysis software written in MATLAB (Supplementary Figs. 2, 3). It is difficult to measure the levels of a pair of second messengers in extending thin growth cones with high motility; however, our imaging and post-processing system enabled the simultaneous measurement of cAMP and cGMP, and also cAMP and $\mathrm{Ca}^{2+}$ in extending growth cones. We prepared dissociated cell cultures of dorsal root ganglion (DRG) neurons from embryonic rats (E18-19) expressing two FRET sensors, and performed dual FRET measurements (Supplementary Fig. 4).

A negative correlation between cAMP and cGMP levels in non-stimulated freely extending growth cones was visualized directly by simultaneous imaging (Fig. 1a-c). The rate of axon extension was unaffected by the excitation laser and the expression of the FRET sensors (Supplementary Fig. 5). As expected, these results support previous studies using pharmacological stimuli ${ }^{8,9}$, and indicate that cAMP and cGMP are regulated reciprocally via an endogenous constitutive mechanism. Additionally, in the experiment to acquire images every $20 \mathrm{~s}$, there was no delay in their correlation in freely extending growth cones (Fig. 1d). This negative correlation may be caused from ensembles of the phosphodiesterase family that have cAMP- and cGMP-selective characteristics $^{8,14,15}$. In contrast, there was no correlation between cAMP and $\mathrm{Ca}^{2+}$ (Fig. 1e-g). However, examination of cross correlations using small time windows showed frequent switching behavior from negative to positive and 

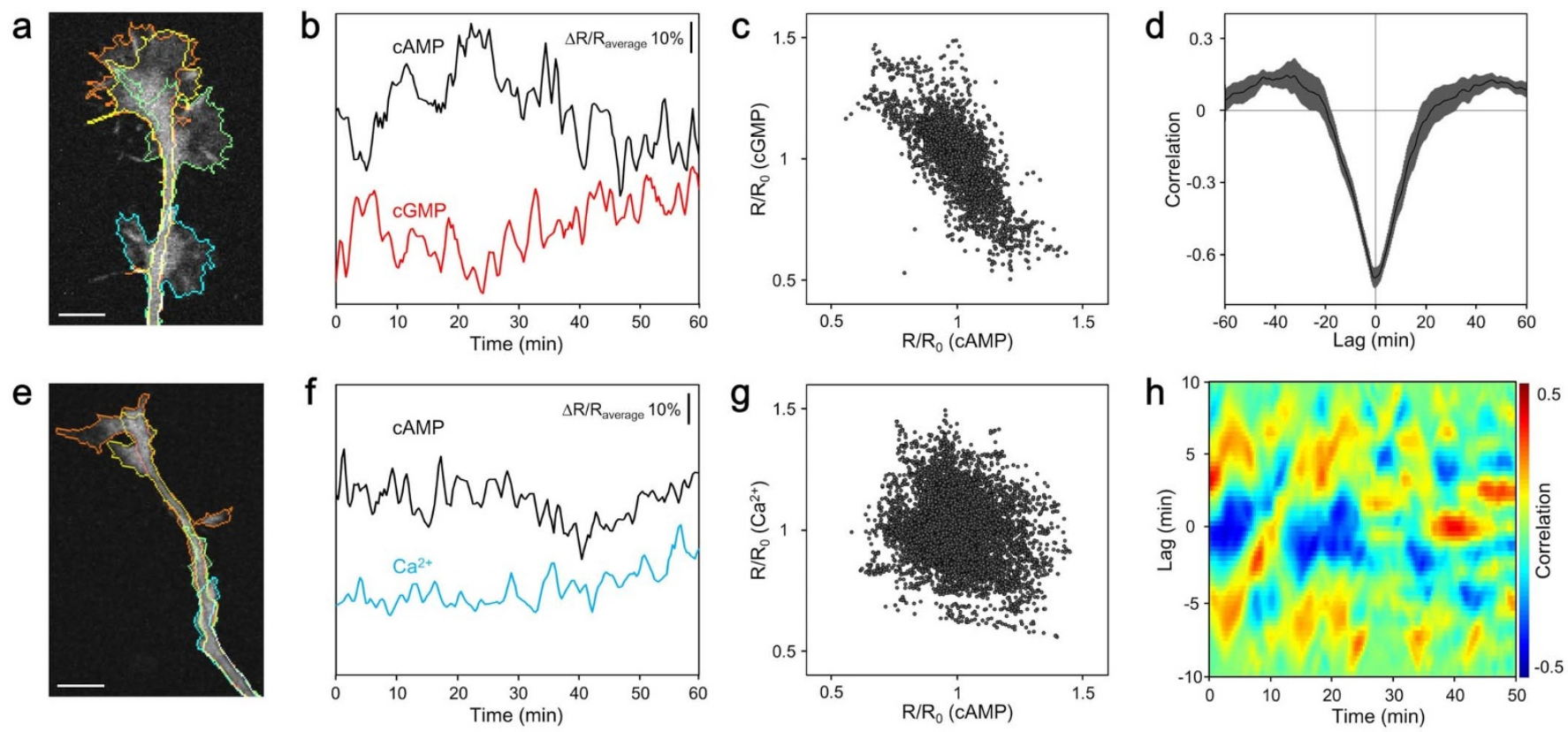

Figure 1 Correlations between cAMP, cGMP, and $\mathrm{Ca}^{2+}$ in growth cones. (a) Representative fluorescence image of an Epac1-camps and red cGES-DE5 co-expressing growth cone. Lines drawn as cyan, green, yellow, and orange indicate the cell edge at $0,20,40$, and 60 min after the onset of image acquisition, respectively. Scale bar represents $10 \mu \mathrm{m}$. (b) Spontaneous changes of $\Delta \mathrm{R} / \mathrm{R}_{\text {average }}$ of ECFP/EYFP (cAMP) and T-Sapphire/dimer2 (cGMP) averaged within the ROIs shown in (a). (c) Negative correlation between cAMP and cGMP levels in the growth cones (correlation coefficient is - 0.71 , 5611 time points from 13 growth cones). (d) Cross correlation function between the spontaneous dynamics of cAMP and cGMP in the growth cones ( $\mathrm{n}=$ 13) showed a negative correlation without delay. Data are presented as means \pm s.e.m. (e) Representative fluorescence image of an Epac1-camps and SapRC2.12 co-expressing growth cone. The colored lines are the same as in (a). Scale bar represents $10 \mu \mathrm{m}$. (f) Representative time course changes in spontaneous $\Delta \mathrm{R} / \mathrm{R}_{\text {average }}$ values of ECFP/EYFP (cAMP) and dimer2/T-Sapphire $\left(\mathrm{Ca}^{2+}\right)$ averaged within the ROIs shown in (e). (g) No correlation between cAMP and $\mathrm{Ca}^{2+}$ levels in the growth cones (correlation coefficient is $-0.19,8529$ time points from 27 growth cones). (h) Heat map showing representative temporal changes in the cross correlation function between the short-term dynamics of cAMP and $\mathrm{Ca}^{2+}$ shown in (f) (see Methods).

vice versa (Fig. 1h). The mechanism underlying this result may be positive feedback regulation of $\mathrm{Ca}^{2+}$ and $\mathrm{cAMP}{ }^{6,7}$.

Spatially asymmetric cAMP and cGMP signals in freely deviating growth cones were visualized directly (Fig. 2a-d). There was no significant change in the right/left cAMP:cGMP ratio (cAMP/ $\left.\mathrm{cGMP}_{\text {right/left }}\right)$ in freely extending growth cones. However, significant

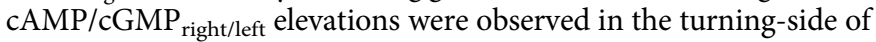
the growth cones at $9.7 \pm 0.5 \mathrm{~min}$ before growth cone angle change. We observed significant increases in the maximum angle change within $15 \mathrm{~min}$ after significant $\mathrm{cAMP} / \mathrm{cGMP}$ right/left elevation (Fig. 2e). Although cytosolic cAMP/cGMP affects growth cone turning responses to netrin $1^{9}$, little is known about the spontaneous dynamics of both cAMP and cGMP. Our result suggests that endogenous changes in CAMP and cGMP affect the growth cone turning angle. Consequently, our result supports a recent study that demonstrated cAMP transients driven by caged compounds steer axons ${ }^{7}$. The observed delay may reflect the signal transduction of cytoskeletal protein reorganization ${ }^{16}$.

We next investigated the relationship between second messengers and axon extension. The cross correlation between cAMP and $\mathrm{Ca}^{2+}$ switched frequently (Fig. 1h). At the timing of switching from pause to extension, the cross correlation switched to negative (Fig. 3 and Supplementary Fig. 6). Although no common correlation was observed before extension, marked negative correlations were observed at the onset of and after extension. The rate of axon outgrowth is reportedly inversely related to the frequency of endogenous $\mathrm{Ca}^{2+}$ transients in growth cones ${ }^{17}$. Although we cannot discuss the frequency of endogenous $\mathrm{Ca}^{2+}$ transients because of the long interval used for image acquisition, the dual FRET method, with a 20 -s interval, revealed the relationship of cAMP and $\mathrm{Ca}^{2+}$ to growth cone motility. A mathematical model based on CaMKII/CaN switching has been advocated and involves the upstream regulators, cAMP and
$\mathrm{Ca}^{2+18,19}$. The determinants of the turning response are not only $\mathrm{Ca}^{2+}$ spike amplitudes but also resting $\mathrm{Ca}^{2+}$ levels; thus, it is very important to monitor resting $\mathrm{Ca}^{2+}$ levels to predict the growth cone turning angle.

\section{Discussion}

During formation of specific neural connections, growth cones navigate axons toward their targets via physical and chemical interactions with environmental cues. Chemotactic substances, mechanical barriers, substratum adhesive substances are vital keys for neural circuit formation. Activation of receptors at axonal tips by these cues may generate signals that are transmitted back to the growth cone where they are transduced into changes in its motility.

$\mathrm{Ca}^{2+}$ signal plays a crucial role in axon pathfinding. Asymmetric $\mathrm{Ca}^{2+}$ signal induces activation of CaMKII and CaN; leading to asymmetric cytoskeletal protein reorganization and endocytosis/exocytosis, which steers the growth cone toward attractive guidance cue ${ }^{4}$. cAMP and cGMP are known as the key regulators of cytosolic $\mathrm{Ca}^{2+}$ through $\mathrm{IP}_{3} \mathrm{R}$ and RyR modulation. Previous reports have shown that the growth cone turning responses to attractive guidance cue netrin 1 depends on the ratio of cAMP and cGMP 9 . In this study, membrane permeable analogues of cAMP and cGMP were used for investigating the turning behavior of the axon, and direct measurement of intracellular second messengers has not been done. As such both cAMP and cGMP are key players in axon guidance, however these dynamics in the migrating growth cone are poorly known, especially in non-stimulated and freely extending axons.

Our results describe the correlations between cAMP and cGMP, and also cAMP and $\mathrm{Ca}^{2+}$, between the asymmetric cAMP/cGMP signal and the growth cone deviation angle among cAMP, $\mathrm{Ca}^{2+}$, and axon extension. Simultaneous dual FRET imaging directly revealed their correlation, and is a suitable method to investigate 
a
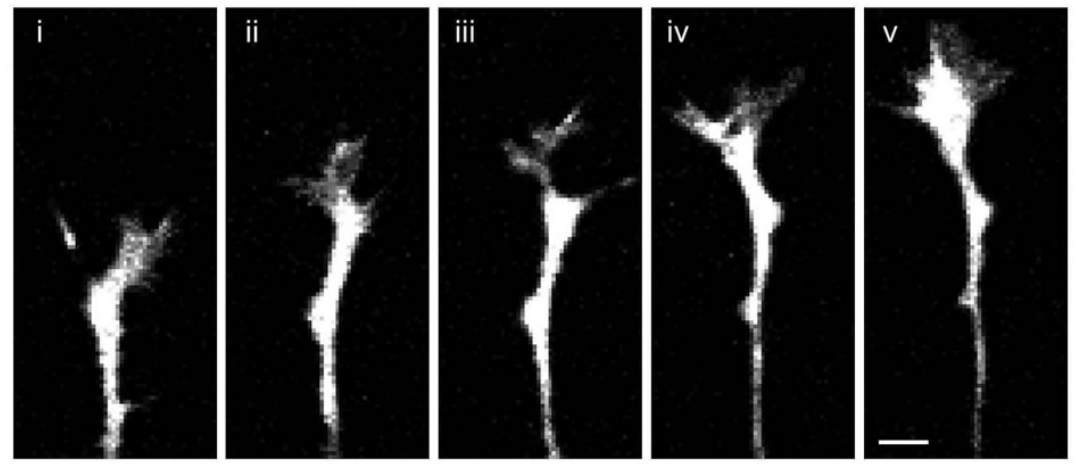

b

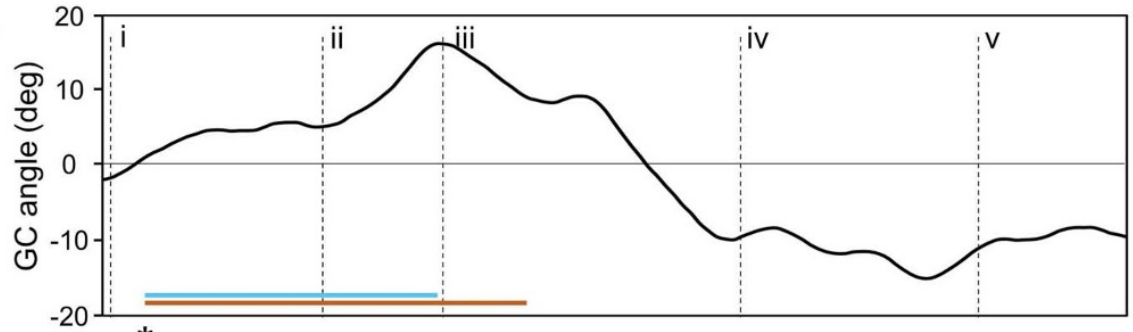

C
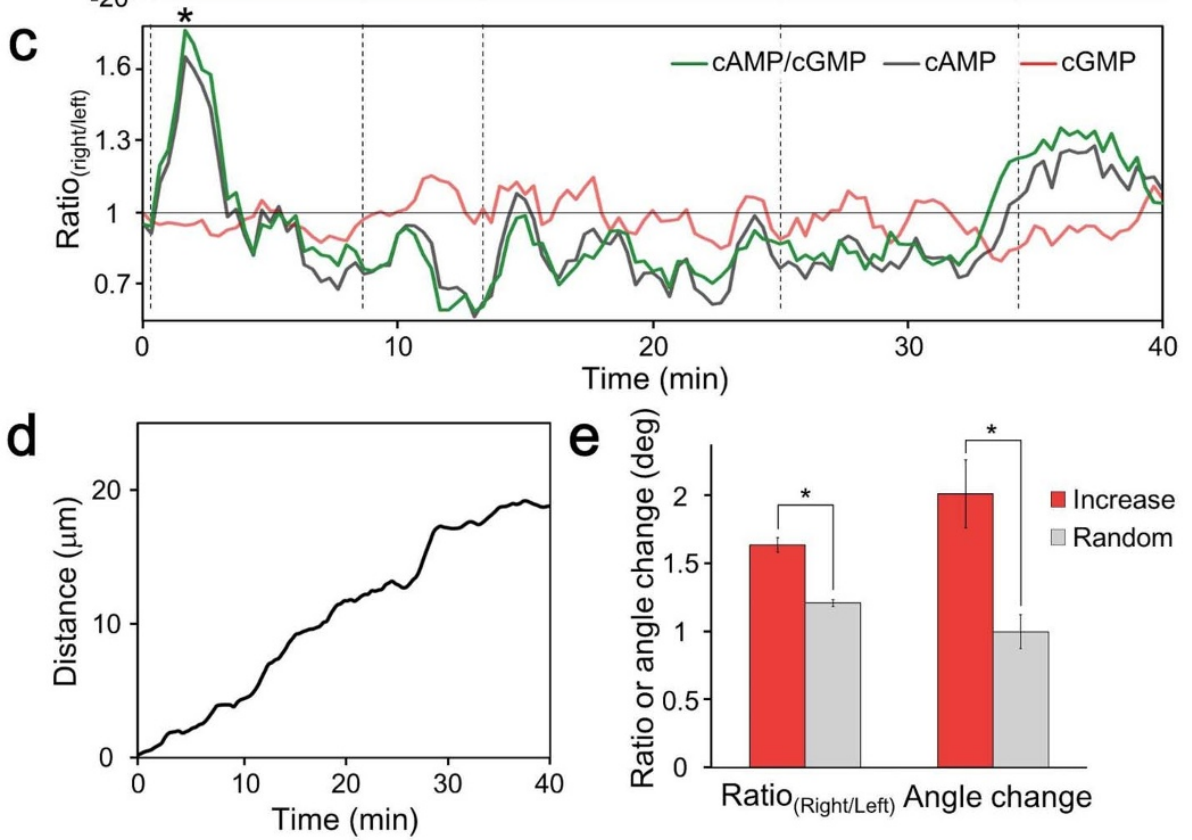

Figure $2 \mid$ Correlations between asymmetric cAMP/cGMP elevation and the deviation angle of growth cones during free extension. (a) Time-lapse fluorescence images of a freely extending growth cone co-expressing Epac1-camps and red cGES-DE5. The Roman numerals (i-v) indicate the time points shown in (b). (b) Time course of growth cone angle changes (see Methods). Right and left turns are defined as positive and negative values, respectively. The Roman numerals correspond those in (a). The orange horizontal line indicates the period when the maximum angle changes were calculated within $15 \mathrm{~min}$ after significant cAMP/cGMP right/left elevation (asterisk). A significant elevation was defined as a change that was 2.58 -fold more

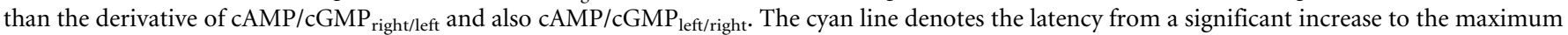
angle change. (c) Spontaneous changes in the right/left ratios of cAMP/cGMP, cAMP, and cGMP (see Methods). (d) Cumulative distance during the observation of the growth cone in Fig. 2a. (e) Freely extending growth cones deviate toward the cAMP/cGMP elevated side. The maximum growth cone angle changes within 15 min after significant cAMP/cGMP elevation at the turning side were higher than in randomly selected cones ( 80 turnings from 14 growth cones). The randomly selected maximum growth cone angle changes were calculated as follows: select a time point from the overall time course and choose the maximum from within 15 min after the time point. Data are presented as means \pm s.e.m. ${ }^{*} P<0.001$.

spatiotemporal crosstalk between two second messenger signals. Additionally, image processing allows us the complex analysis of growth cones with spatially asymmetric signal transduction cascades.

\section{Methods}

Cell culture. Primary DRG neurons dissected from E18 rats (Charles river) were dissociated with $1 \mathrm{mg} / \mathrm{mL}$ trypsin (Sigma-Aldrich) in phosphate-buffered saline. All animal procedures were approved by the Ethics Committee of Keio University (permit number, 09106-(1)). The dissociated cells were resuspended in Neurobasal medium without phenol red (Life Technologies) supplemented with 2\% B27 (Life Technologies) and $50 \mathrm{ng} / \mathrm{mL}$ nerve growth factor (Alomone Labs). The cells were transfected with plasmids coding Epac1-camps and red cGES-DE5, and also Epac1camps and SapRC2.12 by electroporation using Neon (Life Technologies). The cells were plated on a dish coated with $0.01 \mathrm{mg} / \mathrm{mL}$ poly-D-lysine (Life Technologies). The cells were used after a minimum of $24 \mathrm{~h}$ in culture.

Dual FRET measurements. Epacl-camps is fluorescent indicator for cAMP based on the cAMP-binding domain of Epac and exhibits FRET that decrease on addition of $\mathrm{cAMP}^{13}$. Red cGES-DE5 is cGMP sensor based on cGMP binding domain from a 

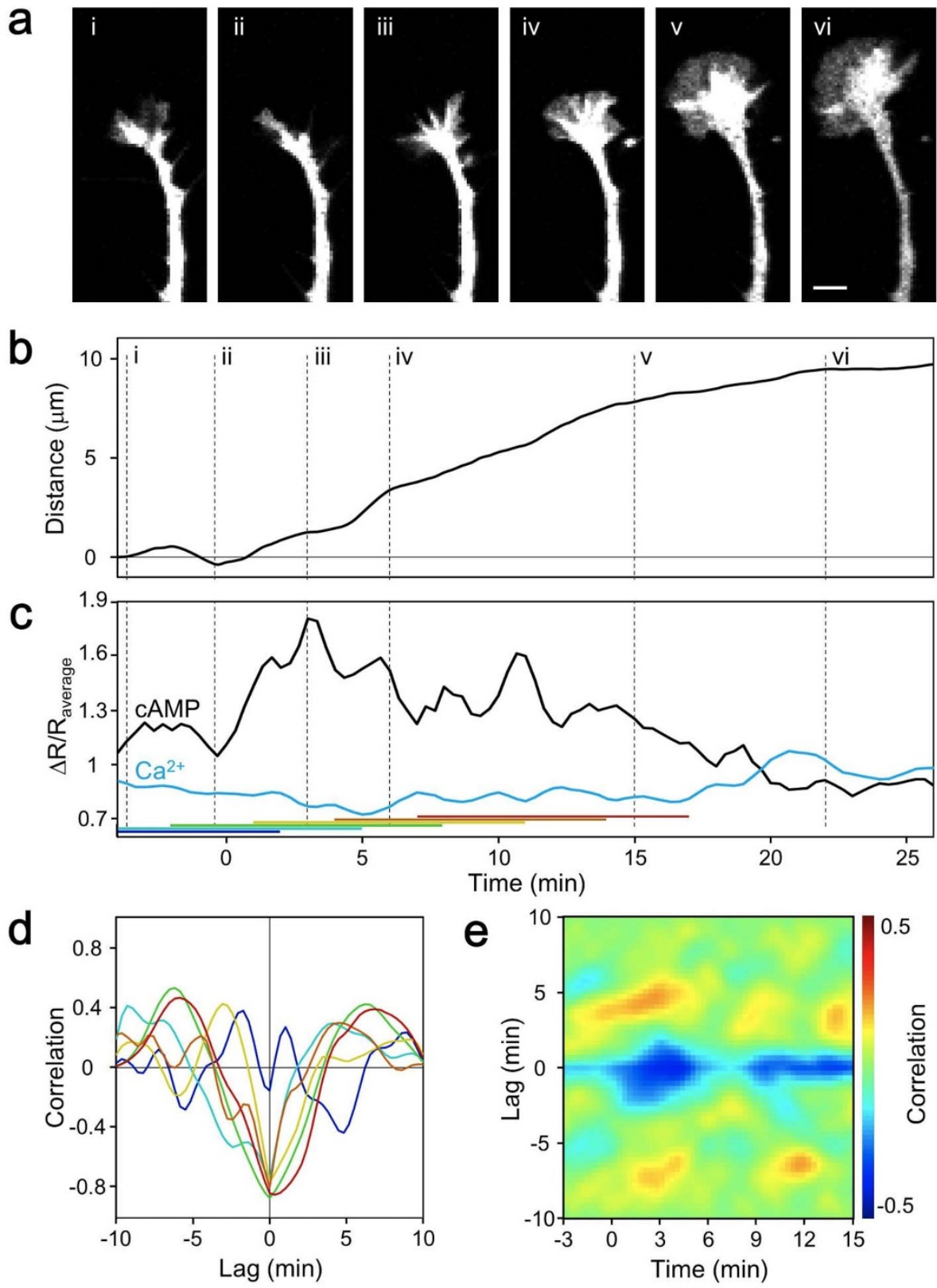

Figure 3 Correlations between cAMP, $\mathrm{Ca}^{2+}$, and extension in freely extending growth cones. (a) Time-lapse fluorescence images of a freely extending growth cone co-expressing Epac1-camps and SapRC2.12 at the time points shown in (b). The Roman numerals (i-vi) indicate the time points shown in (b). Scale bars represent $5 \mu \mathrm{m}$. (b) Cumulative distance during observation of the growth cone in Fig. 3a. The Roman numerals correspond to those in (a).

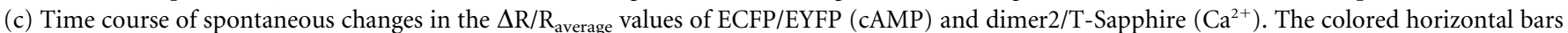
indicate the 10-min time windows used to calculate the correlations shown in (d). The blue, cyan, and other colored bars denote before extension, start of extension, and after extension, respectively. Cross correlations between cAMP and $\mathrm{Ca}^{2+}$ become negative at the change from pause to extension.

(d) Temporal changes in the cross correlation function between the short-term dynamics of cAMP and $\mathrm{Ca}^{2+}$ before and after extension. The colors of the lines indicate the timing of the cross correlation calculations denoted in (c). (e) Heat map showing the averaged sequential time course of the cross correlation function between the short-term dynamics of cAMP and $\mathrm{Ca}^{2+}$. The growth cones started to extend at 0 min $(15$ extensions from 11 growth cones). The start of extension was defined as when the growth cones extended $5 \mu \mathrm{m}$ in $10 \mathrm{~min}$.

phosphodiesterase 5 and also exhibits FRET as Epac1-camps ${ }^{11}$. SapRC2.12 is $\mathrm{Ca}^{2+}$ indicator constructed by fusion of T-Sapphire, CaM, M13 fragment from myosin light chain kinase, and dimer2. SapRC2.12 exhibits FRET that increase upon increase in $\mathrm{Ca}^{2+11}$.

Dual FRET imaging experiments were performed using a laser scanning confocal microscope system (Olympus) mounted on an inverted microscope (Olympus) with a $\times 100$ oil immersion objective. Both donors of the FRET sensors were simultaneously excited using a diode laser at $405 \mathrm{~nm}$, and the excitation was split using a beam splitter 20/80, the emission was split using 3 dichroic mirrors (490, 510, and $560 \mathrm{~nm}$ ), and the emissions were acquired using 2 grating-based detectors (460-490 $\mathrm{nm}$ and $490-510 \mathrm{~nm}$ ) and 2 filter based detectors (535-565 nm and 560-660 nm). Fluorescence images were acquired every
$20 \mathrm{~s}$. Neurons were maintained at $37^{\circ} \mathrm{C}$ in a humidified atmosphere of $5 \% \mathrm{CO}_{2}$ (Tokai Hit).

Image processing. Images were analyzed using original image processing and analysis software written in MATLAB (MathWorks). Acquired images were smoothed using a $3 \times 3$ median filter to reduce noise and the background was subtracted. To obtain the fluorescence signal of each region, ROIs were calculated as described in Supplementary Fig. 2. To obtain the fluorescence signal of each fluorescent protein, an unmixing processing was applied to the fluorescence signals averaged within the ROIs ${ }^{11}$. Finally, the FRET signals were calculated from the unmixed fluorescence ROI-by-ROI. The growth cone angle and movement were also calculated as described in Supplementary Fig. 3. 
Statistical analysis. Cross correlations and all statistical analyses were performed using MATLAB (MathWorks). To investigate the correlation between CAMP and $\mathrm{Ca}^{2+}$, we calculated the temporal changes in cross correlation between the short-term dynamics of cAMP and $\mathrm{Ca}^{2+}$. cAMP and $\mathrm{Ca}^{2+}$ levels in 10-min time windows normalized to their average were used as input for the cross correlation calculations. Significant differences were determined using Student's $t$-test. ${ }^{*} P<0.001$.

1. Tessier-lavigne, M. \& Goodman, C. S. The molecular biology of axon guidance. Science 274, 1123-1133 (1996)

2. Song, H. \& Poo, M. The cell biology of neuronal navigation. Nat. Cell Biol. 3 , E81-8 (2001).

3. Gomez, T. M. \& Zheng, J. Q. The molecular basis for calcium-dependent axon pathfinding. Nat. Rev. Neurosci. 7, 115-25 (2006).

4. Tojima, T., Hines, J. H., Henley, J. R. \& Kamiguchi, H. Second messengers and membrane trafficking direct and organize growth cone steering. Nat. Rev. Neurosci. 12, 191-203 (2011).

5. Zheng, J. Q., Felder, M., Connor, J. A. \& Poo, M. Turning of nerve growth cones induced by neurotransmitters. Nature 368, 140-144 (1994).

6. Gorbunova, Y. V. \& Spitzer, N. C. Dynamic interactions of cyclic AMP transients and spontaneous $\mathrm{Ca}^{2+}$ spikes. Nature 418, 93-6 (2002).

7. Nicol, X., Hong, K. P. \& Spitzer, N. C. Improved molecular toolkit for cAMP studies in live cells. Proc. Natl. Acad. Sci. USA 108, 13776-81 (2011).

8. Shelly, M. et al. Local and long-range reciprocal regulation of cAMP and cGMP in axon/dendrite formation. Science 327, 547-52 (2010).

9. Nishiyama, M. et al. Cyclic AMP/GMP-dependent modulation of Ca2 + channels sets the polarity of nerve growth-cone turning. Nature 423, 990-995 (2003).

10. Giepmans, B. N. G., Adams, S. R., Ellisman, M. H. \& Tsien, R. Y. The fluorescent toolbox for assessing protein location and function. Science 312, 217-24 (2006).

11. Niino, Y., Hotta, K. \& Oka, K. Simultaneous live cell imaging using dual FRET sensors with a single excitation light. PloS one 4, e6036 (2009).

12. Miyawaki, A. Development of probes for cellular functions using fluorescent proteins and fluorescence resonance energy transfer. Annu. Rev. Biochem. 80, 357-73 (2011).

13. Nikolaev, V. O., Bünemann, M., Hein, L., Hannawacker, A. \& Lohse, M. J. Novel single chain cAMP sensors for receptor-induced signal propagation. J. Biol. Chem. 279, 37215-8 (2004).

14. Omori, K. \& Kotera, J. Overview of PDEs and their regulation. Circulation research 100, 309-27 (2007).
15. Mika, D., Leroy, J., Vandecasteele, G. \& Fischmeister, R. PDEs create local domains of cAMP signaling. J. Mol. Cell Cardiol. 52, 323-9 (2012).

16. Wu, Y. I. et al. A genetically encoded photoactivatable Rac controls the motility of living cells. Nature 461, 104-8 (2009).

17. Gomez, T. M. \& Spitzer, N. C. In vivo regulation of axon extension and pathfinding by growth-cone calcium transients. Nature 397, 350-355 (1999).

18. Wen, Z., Guirland, C., Ming, G.-L. \& Zheng, J. Q. A CaMKII/calcineurin switch controls the direction of $\mathrm{Ca}^{2+}$-dependent growth cone guidance. Neuron 43, 835-46 (2004).

19. Forbes, E. M., Thompson, A. W., Yuan, J. \& Goodhill, G. J. Calcium and cAMP levels interact to determine attraction versus repulsion in axon guidance. Neuron 74, 490-503 (2012)

\section{Acknowledgments}

We thank Martin J. Lohse for providing the plasmid Epac1-camps. This work was supported by Grants-in-Aid for Scientific Research (B) (21300112) and Strategic Research Foundation Grant-aided Project for Private Universities from Ministry of Education Culture, Sport, Science, and Technology, Japan (MEXT), 2008-2012, (S0801008).

\section{Author contributions}

T.K., K.H. and K.O. designed the experiments. T.K. performed the experiments and analyzed the data. F.N. assisted with the imaging. T.K. and K.O. wrote the manuscript. K.O supervised.

\section{Additional information}

Supplementary information accompanies this paper at http://www.nature.com/ scientificreports

Competing financial interests: The authors declare no competing financial interests.

How to cite this article: Kobayashi, T., Nagase, F., Hotta, K. \& Oka, K. Crosstalk between Second Messengers Predicts the Motility of the Growth Cone. Sci. Rep. 3, 3118; DOI:10.1038/srep03118 (2013).

(c) (i) $\odot$ This work is licensed under a Creative Commons AttributionNonCommercial-NoDerivs 3.0 Unported license. To view a copy of this license, visit http://creativecommons.org/licenses/by-nc-nd/3.0 